\section{Prevalência e fatores de risco para asma em adolescentes de 13 a 14 anos do Município de Cuiabá, Mato Grosso, Brasil}

\author{
Asthma prevalence and risk factors in adolescents \\ 13 to 14 years of age in Cuiabá, Mato Grosso \\ State, Brazil
}

\author{
1 Instituto de Saúde Coletiva \\ Universidade Federal de \\ Mato Grosso, Cuiabá, Brasil. \\ 2 Faculdade de Medicina \\ Universidade Federal de \\ Mato Grosso, Cuiabá, Brasil. \\ Correspondência \\ S. C. B. M. P. Jucá \\ Instituto de Saúde Coletiva, \\ Universidade Federal de \\ Mato Grosso. \\ Rua 44 319, Cuiabá, MT \\ 78068-505, Brasil. \\ mscjuca@gmail.com
}

\begin{abstract}
The objective of this study was to estimate the prevalence of active asthma and identify associated risk factors in adolescents 13 to 14 years of age in Cuiabá, Mato Grosso State, Brazil. A crosssectional population-based and a subsequently a nested case-control study was conducted in 2008 using the protocol of the International Study of Asthma and Allergies in Childhood (ISAAC). Risk factor analysis used the chi-square test, Fisher's exact test, and binary logistic regression. Variables with $p<0.20$ in the bivariate analysis entered the logistic regression model, with six variables remaining in the final model $(p<0.05)$. Prevalence of active asthma was 19.1\%. Risk factors for active asthma $(p=0.01)$ were: smoking during pregnancy, exclusive breastfeeding $<6$ months, family income $<3$ minimum wages, female gender, pets currently in the home, and absence of fish in the diet. Prevalence of active asthma was high, but with a significant reduction compared to 1998.
\end{abstract}

Asthma; Adolescent; Risk Factors
Sileyde Cristiane B. Matos Póvoas Jucá 1

Olga Akiko Takano 1

Lillian Sanchez Lacerda Moraes 2

Lenir Vaz Guimarães 1

\section{Introdução}

A asma é uma das doenças crônicas mais comuns na infância e o aumento de sua prevalência tem sido motivo de vários estudos epidemiológicos 1 .

Segundo o informe do Global Initiative for Asthma, existem atualmente 300 milhões de pessoas com esta doença no mundo. O custo econômico da asma é considerável, tanto em termos diretos (como internações hospitalares e custo de produtos farmacêuticos) como indiretos (tais como tempo de trabalho perdido, absenteísmo escolar e morte prematura) 2 .

Com o aumento projetado da proporção da população mundial urbana de $45 \%$ para $59 \%$ em 2025, ocorrerá aumento substancial do número de pacientes com asma no mundo nas próximas duas décadas. Estima-se que haverá incremento da ordem de mais de 100 milhões de casos de asma em 20252 .

O International Study of Asthma and Allergies in Childhood (ISAAC) foi idealizado para maximizar o valor da pesquisa epidemiológica em asma e doenças alérgicas na infância, empregando-se método padronizado de amostragem, questionário escrito e vídeo questionário, capaz de facilitar estudos colaborativos, comparar dados obtidos e estimar a prevalência desta doença em âmbito mundial 3,4.

Alguns fatores têm sido identificados como risco para o desenvolvimento de asma e doenças atópicas tais como: atopia familiar, exposição ao 
fumo, ausência de aleitamento materno exclusivo nos primeiros quatro meses de vida, hábitos alimentares e excesso de peso corporal 5,6,7.

O aumento da prevalência da obesidade na população tem coincidido, mundialmente, com o aumento da prevalência de asma. Embora o mecanismo dessa possível associação ainda seja desconhecido, muitas hipóteses têm sido propostas para explicá-la: desenvolvimento de um estado pró-inflamatório, alteração da mecânica ventilatória, influências hormonais, genéticas e neurogênicas 2,7,8,9.

Este estudo tem como objetivo estimar a prevalência de asma ativa e de sintomas relacionados e identificar possíveis fatores de risco associados à doença em adolescentes de 13 a 14 anos, residentes na cidade de Cuiabá, Mato Grosso, Brasil.

\section{Método}

O estudo foi de corte transversal de base populacional, sendo posteriormente aninhado ao mesmo um estudo tipo caso-controle, realizado no período de maio a dezembro de 2008.

A casuística deste estudo foi composta de adolescentes na faixa etária de 13 anos e 1 dia até 14 anos 11 meses e 29 dias de vida, ambos os sexos e matriculados no ensino fundamental da rede pública e particular do Município de Cuiabá.

Foram excluídas da pesquisa escolas localizadas na zona rural de Cuiabá devido à distância e dificuldade de acesso, adolescentes gestantes, portadores de deficiência física e/ou mental e portadores de doenças crônicas incapacitantes.

Os adolescentes que participaram foram selecionados com base na distribuição das escolas de Cuiabá (amostragem aleatória sistemática) fornecida pelas Secretarias Municipal e Estadual de Educação e também estratificados por tipo de escola (pública e particular), zona (norte, sul, leste, oeste e centro) e séries (sétima e oitava).

Respeitando a proporção conforme o tipo de escola (uma particular para cada três públicas sorteadas), foram selecionadas aleatoriamente escolas com maior número de alunos, até que o número mínimo da amostra estivesse completo.

O tamanho da amostra do estudo transversal foi o preconizado pela metodologia do ISAAC, com uma amostra mínima de 3 mil adolescentes e um número mínimo de 10 escolas 3,4.

Para o cálculo da amostra do estudo casocontrole foi utilizado o programa Epi Info versão 3.5.1 (Centers for Disease Control and Prevention, Atlanta, Estados Unidos), considerando um alfa de $5 \%$, uma prevalência populacional de as- ma estimada em $20 \%$, com um fator de proteção de $50 \%(\mathrm{OR}=0,5)$ e um poder de teste de $80 \%$, sendo obtida uma amostra de 217 casos (asmáticos ativos) e 434 controles (não asmáticos).

Para o cálculo da prevalência de asma ativa, utilizou-se como definição de caso aqueles que responderam afirmativamente a questão número dois do questionário escrito do ISAAC "presença de sibilos nos últimos 12 meses".

Foi acrescentada, a questão "alguma vez na vida você teve bronquite?” para comparar com os dados existentes de Amorim \& Daneluzzi 10, passando a ser denominado questionário escrito modificado. Foi utilizado ainda o questionário complementar, modificado para atender as peculiaridades regionais de Cuiabá, visando avaliar possíveis fatores de risco associados a asma, o qual foi denominado questionário complementar modificado.

Antes de aplicar o questionário escrito modificado e questionário complementar modificado do ISAAC, foram realizadas reuniões com os pais e diretores das escolas, ficando estabelecido que o questionário escrito modificado seria aplicado e respondido em sala de aula pelos próprios adolescentes e o questionário complementar modificado seria levado para seus pais/responsáveis para preenchimento domiciliar e devolução na diretoria.

Somente os adolescentes do estudo casocontrole foram submetidos à antropometria para avaliação do índice de massa corpórea. A avaliação antropométrica foi realizada pela pesquisadora principal e por dois outros examinadores previamente treinados, com a aferição do peso e da estatura com os indivíduos descalços e com o mínimo de roupa, as medidas obtidas eram colocadas em uma ficha individual, juntamente com dados sobre maturação puberal. Foi utilizada balança digital com capacidade de $150 \mathrm{~kg}$ e sensibilidade de 100g. A estatura foi obtida com os adolescentes em posição ereta, pés unidos e em paralelo, por estadiômetro. Para a classificação do estado nutricional, o IMC $\left(\mathrm{kg} / \mathrm{m}^{2}\right)$ foi calculado e plotado na curva referenciada pelo Centers for Disease Control and Prevention 11.

Os dados relativos ao questionário escrito modificado, questionário complementar modificado e antropometria foram digitados em duplicata no programa SPSS, versão 15 (SPSS Inc., Chicago, Estados Unidos). Para análise dos dados e cálculo das prevalências e respectivos intervalos de confiança utilizaram-se os pacotes estatísticos SPSS e Epi Info versão 3.5.1. Para o cálculo das associações entre asma ativa e variáveis explicativas foi utilizado o teste de qui-quadrado de Pearson, nível de significância menor que 0,05. $\mathrm{O}$ efeito das variáveis explicativas na ocorrência 
de asma ativa foi avaliado pelo cálculo de odds ratio, intervalo de 95\% de confiança (IC95\%). As variáveis que apresentavam $\mathrm{p}<0,20$ na análise bivariada foram incluídas no modelo de regressão logística (Backward: Wald). No modelo final de regressão logística múltipla as variáveis com $\mathrm{p}$ $<0,05$ foram consideradas suficientes para estabelecer significância estatística.

O estudo foi submetido ao Comitê de Ética em Pesquisa do Hospital Universitário Júlio Müller e aprovado em 14 de novembro de 2007, sob o protocolo $n^{\circ}$. 436/CEP/HUJM/07. A pesquisa e o Termo de Consentimento Livre e Esclarecido foram explicados durante as reuniões dos pais com os diretores das escolas. Somente foram incluídos na análise, os adolescentes cujos pais/responsáveis concordaram e assinaram o termo. Nenhuma das escolas selecionadas recusou participar e seus diretores também assinaram o termo. Todos os pacientes identificados com presença de asma ativa e/ou sobrepeso/obesidade receberam uma carta com os resultados e recomendação de avaliação médica no Hospital Universitário Júlio Müller ou pelo médico da família.

\section{Resultados}

Foram distribuídos 3.500 questionários, com taxa de retorno de 98\% (3.430). Destes, foram excluídos 88 questionários por erro de preenchimento ou preenchimento incompleto, totalizando uma amostra válida de 3.342 questionários (95,5\%). A distribuição destes adolescentes segundo idade, sexo, tipo de escola e região é mostrada na Tabela 1 .

No estudo caso-controle totalizaram 590 questionários válidos, sendo 198 asmáticos ativos e 392 não asmáticos, com uma perda de 8,8\% e $9,7 \%$ respectivamente para cada grupo.

A prevalência dos sintomas de asma em relação ao sexo, bem como a prevalência de diagnóstico médico de asma e bronquite estão apresentadas na Tabela 2. Observou-se que todas as prevalências encontradas foram significantemente maiores no sexo feminino, exceto a prevalência do diagnóstico médico de bronquite, que foi superior nos adolescentes masculinos. Não houve diferença estatística significante entre os gêneros para as prevalências de 4 ou mais crises por semana nos últimos 12 meses e de diagnóstico médico de bronquite.

Na Tabela 3, os resultados obtidos para a pergunta "presença de sibilos nos últimos 12 meses" (asma ativa) e "bronquite diagnosticada por médico" mostram uma redução significante para a prevalência de asma ativa e bronquite diagnosticada por médico.
Na análise bivariada para avaliação dos fatores de risco relacionados à presença de asma ativa, observou-se associação com os seguintes fatores: sexo feminino, renda familiar menor que três salários-mínimos, mãe asmática e ambos os pais asmáticos, tabagismo na gestação e no primeiro ano vida, exposição aos animais no domicílio, tanto no primeiro ano de vida do adolescente como no presente, presença de umidade em casa ocorrida no primeiro ano de vida, ausência de peixe na dieta e tempo de aleitamento materno $<6$ meses exclusivo ou não (Tabela 4 ).

$\mathrm{Na}$ análise multivariada, utilizando-se como variável dependente a presença de asma ativa, seis variáveis mantiveram-se como fatores de risco associados ao desenvolvimento de asma: tabagismo na gestação, seio materno exclusivo $<6$ meses, renda familiar < 3 salários-mínimos, sexo feminino, animais em casa no presente e ausência de peixe na dieta (Tabela 5).

\section{Discussão}

Observou-se uma homogeneidade na distribuição dos adolescentes da amostra, tanto em relação ao gênero dos adolescentes quanto em

Tabela 1

Distribuição das características gerais dos 3.342

adolescentes. Cuiabá, Mato Grosso, Brasil, 2008.

\begin{tabular}{|c|c|c|}
\hline Variáveis & $\mathrm{n}$ & $\%$ \\
\hline \multicolumn{3}{|l|}{ Idade (anos) } \\
\hline 13 & 1.638 & 49,0 \\
\hline 14 & 1.704 & 51,0 \\
\hline \multicolumn{3}{|l|}{ Sexo } \\
\hline Masculino & 1.570 & 47,0 \\
\hline Feminino & 1.772 & 53,0 \\
\hline \multicolumn{3}{|l|}{ Raça/Cor } \\
\hline Parda & 1.421 & 42,5 \\
\hline Branca & 996 & 29,8 \\
\hline Negra & 656 & 19,6 \\
\hline Indígena & 48 & 1,4 \\
\hline Asiática & 28 & 0,8 \\
\hline Ignorada & 193 & 5,8 \\
\hline \multicolumn{3}{|l|}{ Escolas } \\
\hline Públicas & 2.505 & 75,0 \\
\hline Privadas & 837 & 25,0 \\
\hline \multicolumn{3}{|l|}{ Região } \\
\hline Norte & 788 & 23,6 \\
\hline Sul & 791 & 23,7 \\
\hline Leste & 817 & 24,4 \\
\hline Oeste & 946 & 28,3 \\
\hline
\end{tabular}


Prevalência de respostas afirmativas ao questionário escrito modificado do International Study of Asthma and Allergies in Childhood, módulo asma, entre escolares de 13 a 14 anos segundo o sexo. Cuiabá, Mato Grosso, Brasil, 2008.

\begin{tabular}{|c|c|c|c|c|c|c|c|}
\hline \multirow[t]{3}{*}{ Questões } & \multicolumn{4}{|c|}{ Sexo } & \multicolumn{2}{|c|}{ Total $(n=3.342)$} & \multirow[t]{3}{*}{ OR (IC95\%) } \\
\hline & \multicolumn{2}{|c|}{ Masculino $(n=1.570)$} & \multicolumn{2}{|c|}{ Feminino $(n=1.772)$} & \multirow[b]{2}{*}{$\mathbf{n}$} & \multirow[b]{2}{*}{$\%$} & \\
\hline & $\mathbf{n}$ & $\%$ & $\mathbf{n}$ & $\%$ & & & \\
\hline 1. Sibilos alguma vez & 577 & 36,8 & 827 & 46,7 & 1.404 & 42,0 & $1,5(1,3-1,7)$ \\
\hline 2. Sibilos nos últimos 12 meses & 248 & 15,8 & 391 & 22,1 & 639 & 19,1 & $1,5(1,3-1,8)$ \\
\hline \multicolumn{8}{|l|}{ 3. Crises nos últimos 12 meses } \\
\hline$<4$ & 200 & 12,7 & 319 & 18,0 & 519 & 15,5 & $1,5(1,2-1,8)$ \\
\hline 4 ou mais & 37 & 2,4 & 54 & 3,0 & 91 & 2,7 & $1,3(0,8-2,0)$ \\
\hline \multicolumn{8}{|l|}{ 4. Sono prejudicado por chiado } \\
\hline$<1$ noite/semana & 83 & 5,3 & 158 & 8,9 & 241 & 7,2 & $1,7(1,3-2,3)$ \\
\hline 1 ou + noites/semana & 38 & 2,4 & 68 & 3,8 & 106 & 3,2 & $1,6(1,1-2,5)$ \\
\hline 5. Limitação da fala & 38 & 2,4 & 79 & 4,5 & 117 & 3,5 & $1,9(1,2-2,8)$ \\
\hline 6. Diagnóstico médico de asma & 107 & 6,8 & 175 & 9,9 & 282 & 8,4 & $1,5(1,2-1,9)$ \\
\hline 7. Sibilo aos exercícios físicos & 241 & 15,4 & 338 & 19,1 & 579 & 17,3 & $1,3(1,1-1,6)$ \\
\hline 8. Tosse seca sem infecção & 602 & 38,3 & 889 & 50,2 & 1.491 & 44,6 & $1,6(1,4-1,9)$ \\
\hline 9. Diagnóstico médico de Bronquite & 362 & 23,1 & 401 & 22,6 & 763 & 22,8 & $1,0(0,8-1,1)$ \\
\hline
\end{tabular}

IC95\%: intervalo de 95\% de confiança; OR: odds ratio.

Categoria de referência: sexo masculino $(\mathrm{OR}=1)$.

Tabela 3

Prevalência de asma ativa e diagnóstico médico de bronquite, entre adolescentes de 13 a 14 anos, segundo o ano de aplicação do questionário escrito modificado do International Study of Asthma and Allergies in Childhood - módulo asma. Cuiabá, Mato Grosso, Brasil, 2008.

\begin{tabular}{|c|c|c|c|c|c|c|}
\hline & \multicolumn{2}{|c|}{1998 * } & \multicolumn{2}{|c|}{2008} & \multirow[t]{2}{*}{$\chi^{2}$} & \multirow[t]{2}{*}{ Valor de $\mathrm{p}$} \\
\hline & $n$ & $\%$ & $\mathbf{n}$ & $\%$ & & \\
\hline \multicolumn{7}{|c|}{ Asma ativa } \\
\hline Sim & 744 & 21,2 & 639 & 19,1 & 4,6 & 0,01 \\
\hline Não & 2.765 & 78,8 & 2.703 & 80,9 & & \\
\hline \multicolumn{7}{|c|}{ Diagnóstico de bronquite } \\
\hline Sim & 926 & 26,4 & 762 & 22,8 & 11,9 & 0,01 \\
\hline Não & 2.583 & 73,6 & 2.580 & 77,2 & & \\
\hline
\end{tabular}

* Amorim \& Daneluzzi 10 .

relação à localização das escolas. Um número representativo da população do estudo para cada uma das quatro principais regiões do Município de Cuiabá, bem como um número equilibrado de adolescentes de ambos os gêneros, minimizaram os possíveis vieses de seleção.

A análise da raça/cor mostrou predomínio de adolescentes de raça/cor parda, evidenciando o padrão de miscigenação não só da população brasileira como da região matogrossense, onde existe uma importante contribuição da raça negra e indígena na formação da população 12 .
Os resultados mundiais, observados nas Fases I e III do ISAAC, evidenciaram tendência de estabilização e até de redução da prevalência de asma em adolescentes, em algumas regiões como a Oceania. Ao mesmo tempo, em algumas regiões como África, Índia, América Latina, Europa do Norte e Europa Oriental, o que se observou foi aumento da prevalência de asma 13,14.

No Brasil, apesar da prevalência de asma mostrar tendência à diminuição, é ainda a mais alta dentre os países da América Latina, com valores médios de $20 \%$, variando de uma região para ou- 
Distribuição de 590 escolares de 13-14 anos (casos = 198 asmáticos, controles = 392 não asmáticos) segundo características sociodemográficas e fatores de risco associados.

\begin{tabular}{|c|c|c|c|c|c|c|}
\hline \multirow[t]{2}{*}{ Variáveis } & \multicolumn{2}{|c|}{ Casos $(n=198)$} & \multicolumn{2}{|c|}{ Controles $(n=392)$} & \multirow[t]{2}{*}{ OR (IC95\%) } & \multirow[t]{2}{*}{ Valor de $p$} \\
\hline & $\mathbf{n}$ & $\%$ & $\mathbf{n}$ & $\%$ & & \\
\hline Sexo feminino & 136 & 68,7 & 198 & 50,5 & $2,1(1,5-3,1)$ & 0,01 \\
\hline Renda $<3$ salários mínimos & 130 & 65,7 & 196 & 50,0 & $1,0(1,3-2,7)$ & 0,01 \\
\hline Ambos os pais asmáticos & 13 & 6,6 & 9 & 2,2 & $3,0(1,3-7,1)$ & 0,01 \\
\hline Tabagismo na gestação & 46 & 23,3 & 43 & 11,0 & $2,5(1,6-3,9)$ & 0,01 \\
\hline Tabagismo no primeiro ano & 54 & 27,3 & 71 & 18,1 & $1,7(1,1-2,5)$ & 0,01 \\
\hline Animais em casa no primeiro ano & 59 & 29,8 & 67 & 17,1 & $2,0(1,4-3,1)$ & 0,01 \\
\hline Animais em casa no presente & 70 & 35,4 & 97 & 24,7 & $1,6(1,1-2,4)$ & 0,01 \\
\hline Aleitamento materno $<6$ meses * & 76 & 46,9 & 98 & 28,3 & $2,2(1,5-3,3)$ & 0,01 \\
\hline Aleitamento materno exclusivo $<6$ meses * & 131 & 80,9 & 206 & 59,5 & $2,9(1,8-4,5)$ & 0,01 \\
\hline Ausência de peixe na dieta & 60 & 30,3 & 75 & 19,1 & $1,8(1,2-2,7)$ & 0,01 \\
\hline Mãe asmática & 20 & 10,1 & 19 & 4,8 & $2,2(1,1-4,2)$ & 0,02 \\
\hline Umidade em casa no primeiro ano & 46 & 23,2 & 62 & 15,8 & $1,6(1,1-2,5)$ & 0,03 \\
\hline Ausência de vegetais crus na dieta & 55 & 27,8 & 80 & 20,4 & $1,5(1,0-2,2)$ & 0,05 \\
\hline Umidade em casa no presente & 38 & 19,2 & 50 & 12,8 & $1,6(1,0-2,6)$ & 0,05 \\
\hline Parto cesáreo & 120 & 60,6 & 206 & 52,6 & $1,4(1,0-2,0)$ & 0,07 \\
\hline Peso ao nascer $<2.500 \mathrm{~g}$ & 36 & 18,2 & 50 & 12,8 & $1,5(0,9-2,4)$ & 0,08 \\
\hline Sobrepeso/Obesidade & 30 & 15,2 & 42 & 10,7 & $1,5(0,9-2,5)$ & 0,12 \\
\hline Ausência de carne na dieta & 21 & 10,6 & 27 & 4,6 & $1,6(0,9-2,9)$ & 0,15 \\
\hline Pai asmático & 17 & 8,6 & 21 & 5,4 & $1,7(0,8-3,2)$ & 0,16 \\
\hline Ausência de frutas na dieta & 17 & 8,6 & 23 & 5,9 & $1,5(0,8-2,9)$ & 0,23 \\
\hline Ausência de vegetais cozidos na dieta & 26 & 13,1 & 44 & 11,2 & $1,2(0,7-2,0)$ & 0,50 \\
\hline Cor parda/negra & 152 & 76,8 & 311 & 79,3 & $1,2(0,8-1,8)$ & 0,52 \\
\hline Tabagismo no presente & 47 & 23,7 & 84 & 21,4 & $1,1(0,8-1,7)$ & 0,53 \\
\hline Prematuridade & 25 & 12,6 & 43 & 11,0 & $1,2(0,7-2,0)$ & 0,59 \\
\hline Ausência de suco de frutas na dieta & 14 & 7,1 & 22 & 5,6 & $1,3(0,6-2,6)$ & 0,59 \\
\hline Presença de refrigerante na dieta & 196 & 99,0 & 387 & 98,7 & $1,3(0,2-6,5)$ & 1,00 ** \\
\hline
\end{tabular}

* Nas variáveis aleitamento materno (> 6 meses ou exclusivo), o número de casos $(n=162)$ e controles $(n=346)$ foram diferentes;

** Teste exato de Fisher.

tra15. Os resultados da Fase III do ISAAC no Brasil revelaram taxas de prevalência de asma ativa variando de 11,8\% em Nova Iguaçu (Rio de Janeiro) a 30,5\% em Vitória da Conquista (Bahia) 15 . Em Tangará da Serra (Mato Grosso) a prevalência encontrada, entre adolescentes, foi de $15,9 \% 16$ e em Alta Floresta (Mato Grosso) foi de 12,4\% 17. A prevalência de asma ativa encontrado no presente estudo foi de $19,1 \%$, estando dentre os valores médios encontrados no Brasil.

Estilo de vida ocidentalizado, urbanização e aumento da densidade populacional são aspectos utilizados para explicar a ocorrência da variabilidade da prevalência entre os diferentes estudos 15 .

A questão “asma alguma vez na vida”, relatada apenas por 8,4\% dos adolescentes neste estudo, refere-se ao diagnóstico fornecido por um médico. O hábito, algumas vezes do próprio profissional, de utilizar termos mais populares como bronquite, bronquite alérgica, bronquite asmática, bem como a dificuldade dos próprios adolescentes de entenderem a diferença entre asma e bronquite, acabam ocasionando subdiagnóstico de asma, o que pode dificultar medidas mais efetivas para o tratamento e controle desta doença. Amorim \& Daneluzzi 10, na questão de número 6 do módulo asma do questionário escrito do ISAAC ("alguma vez na vida você teve asma") substituíram o termo asma pelo termo bronquite. Para fins de comparação, neste estudo o questionário escrito do ISAAC foi modificado, acrescentando ao módulo asma a questão: "alguma vez na vida você teve bronquite” (diagnóstico médico de bronquite). 
Análise das variáveis do estudo dos escolares de 13-14 anos ( $n=198$ escolares asmáticos, $n=392$ não asmáticos) que entraram no modelo de regressão logística. Cuiabá, Mato Grosso, Brasil, 2008.

\begin{tabular}{|c|c|c|c|c|c|}
\hline Variáveis & OR não ajustado & IC95\% & OR ajustado & IC95\% & Valor de $p$ \\
\hline Tabagismo na gestação * & 2,5 & $1,6-3,9$ & 2,8 & $1,6-4,9$ & 0,01 \\
\hline Seio materno exclusivo $<6$ meses * & 2,9 & $1,8-4,5$ & 2,5 & $1,5-4,4$ & 0,01 \\
\hline Renda familiar $<3$ salários mínimos * & 2,6 & $1,7-4,0$ & 2,1 & $1,4-3,3$ & 0,01 \\
\hline Sexo feminino * & 2,1 & $1,5-3,1$ & 2,5 & $1,6-3,9$ & 0,01 \\
\hline Animais em casa no presente * & 1,6 & $1,1-2,4$ & 1,8 & $1,2-2,9$ & 0,01 \\
\hline Ausência de peixe na dieta * & 1,8 & $1,2-2,7$ & 1,8 & $1,1-3,0$ & 0,01 \\
\hline Ambos os pais asmáticos & 3,0 & $1,3-7,1$ & 1,7 & $0,5-6,0$ & 0,42 \\
\hline Mãe asmática & 2,2 & $1,1-4,2$ & 1,0 & $0,3-3,0$ & 0,74 \\
\hline Pai asmático & 1,7 & $0,9-3,2$ & 0,8 & $0,2-2,8$ & 0,64 \\
\hline Parto cesáreo & 1,4 & $1,0-2,0$ & 1,5 & $1,0-2,3$ & 0,08 \\
\hline Peso de nascimento $<2.500 \mathrm{~g}$ & 1,5 & $0,9-2,5$ & 1,6 & $0,8-2,9$ & 0,15 \\
\hline Aleitamento materno $<6$ meses & 2,2 & $1,5-3,3$ & 1,6 & $1,0-2,6$ & 0,06 \\
\hline Tabagismo no 1ㅇa ano & 1,7 & $1,1-2,5$ & 0,8 & $0,3-2,2$ & 0,63 \\
\hline Umidade em casa no 1ㅇ ano & 1,6 & $1,0-2,5$ & 1,7 & $1,0-2,9$ & 0,07 \\
\hline Umidade em casa no presente & 1,6 & $1,0-2,5$ & 1,5 & $0,8-2,6$ & 0,21 \\
\hline Animais em casa no 1 으 ano & 2,0 & $1,4-3,1$ & 1,3 & $0,7-2,5$ & 0,41 \\
\hline Ausência de carne na dieta & 1,6 & $0,9-2,9$ & 2,2 & $1,0-4,5$ & 0,06 \\
\hline Ausência de vegetais crus na dieta & 1,5 & $1,0-2,0$ & 1,5 & $0,9-2,4$ & 0,12 \\
\hline Sobrepeso/Obesidade & 1,5 & $0,9-2,5$ & 1,4 & $0,8-2,4$ & 0,18 \\
\hline
\end{tabular}

* Com significância estatística.

IC95\%: intervalo de 95\% de confiança; OR: odds ratio.

Comparando os resultados encontrados com os observados por Amorim \& Daneluzzi 10, em 1998, observou-se redução estatisticamente significante na prevalência de asma ativa em um período de dez anos (21,2\% em 1998 versus $19,1 \%$ em 2008), bem como na prevalência do diagnóstico médico de bronquite, que passou de $26,4 \%$ em 1998, para 22,8\%. Solé et al. 15 , comparando a fase I e III do ISAAC, também observaram a tendência de estabilização e até redução da prevalência de asma.

As diferenças entre diagnóstico médico de asma e "sibilos nos últimos 12 meses" encontradas nesse estudo, também ocorreram em outras cidades brasileiras, entre elas a cidade de São Paulo (regiões centro sul e oeste) nos anos de 1995, 1996, 2002 e 2006, onde apenas 10\%, 8,4\%, $10,3 \%$ e $8,9 \%$ dos adolescentes respectivamente, responderam ter recebido o diagnóstico de "asma alguma vez" 18,19. Mallol et al. 20, na América Latina, mostraram essa mesma tendência, sendo a prevalência média de "asma alguma vez" 12,6\% com variação de 5,5\% a 28\%. Os resultados publicados da Fase I - ISAAC evidenciaram prevalência média de 11,3\% para “asma alguma vez", variando de $1,4 \%$ a 30,4\%, com significantes diferenças mesmo dentro de um mesmo país. Na Fase III, no Brasil, a prevalência média de asma diagnosticada por médico foi $13,6 \%$ com valores mais elevados em Belém, Porto Alegre e Caruaru 15.

A questão "sibilos nos últimos 12 meses" assume um papel de grande importância, pois essa limitação de tempo diminui o viés de memória e torna independente o mês do preenchimento do questionário, fazendo com que esta seja a questão de maior sensibilidade e especificidade na definição de casos de asma 4 .

Os dados encontrados neste trabalho são compatíveis com os de outros estudos que mostram ser a prevalência de asma e de sintomas relacionados superior no sexo feminino na adolescência 21,22,23.

Na análise comparativa das variáveis, entre os adolescentes asmáticos ativos (casos) e não asmáticos (controles), o presente estudo também encontrou associação entre o sexo feminino e asma, tanto na análise bivariada como na multivariada.

O impacto do gênero varia consideravelmente da infância para adolescência e idade adulta 24 . O predomínio da asma no sexo feminino pode ser explicado pelos efeitos imunomoduladores dos hormônios sexuais 25,26. Além das diferenças hormonais, os gêneros, também diferem quanto 
ao desenvolvimento e a função pulmonar 26,27 . No sexo masculino há relativa lentidão no desenvolvimento das vias aéreas quando comparado com o aumento dos volumes pulmonares. Já no sexo feminino o crescimento das vias aéreas e o aumento do volume pulmonar são proporcionais, consequentemente com maiores fluxos aéreos, o que é uma vantagem principalmente na sibilância envolvendo infecções virais. Na puberdade, estas diferenças se revertem com a aceleração de todos os índices de função pulmonar no sexo masculino 27.

No presente estudo, encontrou-se um maior risco para asma entre adolescentes com renda familiar mais baixa (menor que três saláriosmínimos), com diferença estatisticamente significativa mantida após análise multivariada. As diferenças de prevalência da asma quando considerados diferentes níveis socioeconômicos mostram resultados controversos 28,29. No Brasil, alguns estudos encontraram prevalência de asma maior em adolescentes de baixo nível socioeconômico 30,31. Entretanto em publicação recente, Solé et al. 32 evidenciaram que a prevalência de asma e seus sintomas são variáveis e independentes do nível socioeconômico.

O aleitamento materno, exclusivo ou não, por menos de seis meses, mostrou ser um fator de risco para o desenvolvimento de asma ativa, com diferença estatística significante, mesmo após análise multivariada.

Bener et al. 33 demonstraram que o aleitamento materno superior a seis meses protege contra o desenvolvimento de asma. A variabilidade do tempo de aleitamento e as diferenças genéticas entre a população estudada influenciam nos resultados dos estudos 34 . Friedman \& Zeiger 35 revisaram os artigos contra e a favor do aleitamento materno na prevenção de doenças alérgicas e concluíram que este deve ser encorajado por pelo menos quatro a seis meses para toda criança, independente de seu risco, ou da história materna positiva para asma.

Após análise multivariada, a presença de tabagismo materno na gestação permaneceu como significante fator de risco para asma ativa. $\mathrm{O}$ tabagismo, em particular o tabagismo materno durante a gestação e primeiro ano de vida, é um significante fator de risco para o subsequente desenvolvimento de asma em crianças 36,37. Níveis elevados de IL-13 foram encontrados em pacientes com asma e pais fumantes, além de correlação positiva entre IL-13 e concentrações séricas de IgE em crianças com asma alérgica 38,39. Estudos recentes mostram o tabagismo, principalmente o materno, como importante fator de risco não apenas no desenvolvimento de asma, como no agravamento dos sintomas 39,40,41.
$\mathrm{Na}$ análise do contato dentro de casa com animais no presente, verificou que a exposição domiciliar atual a animais é um fator de risco significante para o desenvolvimento de asma ativa. Os animais domésticos são considerados fontes de alérgenos intradomiciliares, e a relação entre a exposição aos mesmos na infância e desenvolvimento de sensibilização e sintomas de asma ainda são complexas 42 . Fatores como época da chegada do animal no domicilio, idade da criança à exposição, número de animais, carga alergênica do ambiente e história familiar de atopia podem modular a expressão da sensibilização a esses alérgenos 43,44 .

A ausência de peixe na dieta mostrou ser um fator de risco significante para o desenvolvimento de asma. Recentemente, fatores dietéticos foram associados com a prevalência de asma e atopia 34,45. As dietas com baixos teores de antioxidantes ou ácidos graxos poliinsaturados são consideradas fator de risco para o desenvolvimento de resposta inflamatória associada à asma 46 . Estudos têm demonstrado que a ingestão de frutas frescas em maior quantidade, ricas em flavonóides com suas propriedades antioxidantes, bem como a ingestão de ômega 3 proveniente do óleo de peixe, devido seus efeitos antiinflamatórios, inibe a formação de leucotrienos e prostaglandinas, tendo efeito protetor em relação à asma 34 .

No presente estudo, não houve associação de sobrepeso/obesidade e asma ativa entre os adolescentes. Considerando-se que a prevalência da obesidade é extremamente alta entre crianças e adolescentes que vivem em ambientes urbanos, onde a prevalência de asma também é particularmente alta, se faz necessário conhecer os mecanismos envolvidos nessa relação 9,46.

\section{Conclusão}

A prevalência de asma ativa para o Município de Cuiabá foi alta com redução significante em relação à prevalência encontrada em 1998.

Há necessidade de desenvolver um programa municipal de capacitação profissional em asma que ofereça ao adolescente asmático um atendimento multidisciplinar, com avaliação de médicos, nutricionistas e psicólogos treinados. $\mathrm{O}$ planejamento e execução de programas de educação que visem ao controle da asma, mostrando a importância da alimentação balanceada, do aleitamento materno, da higiene ambiental adequada, poderão contribuir para a melhora na qualidade de vida de crianças e adolescentes portadores dessa enfermidade. 


\section{Resumo}

Estimar a prevalência de asma ativa e identificar possíveis fatores de risco associados em adolescentes de 13 a 14 anos, residentes na cidade de Cuiabá, Mato Grosso, Brasil. Estudo transversal de base populacional sendo posteriormente aninhado a estudo caso-controle, realizado em 2008, utilizando o protocolo do International Study of Asthma and Allergies in Childhood (ISAAC). A análise dos possiveis fatores de risco foi realizada pelo teste do qui-quadrado, teste exato de Fisher e por regressão binária logística. As variáveis com $p<$ 0,20 na análise bivariada entraram no modelo de regressão logística, permanecendo no modelo final seis variáveis $(p<0,05)$. A prevalência de asma ativa foi de $19,1 \%$. Os fatores de risco para asma ativa $(p=0,01)$ foram: tabagismo na gestação, seio materno exclusivo $<6$ meses, renda familiar < 3 salários mínimos, sexo feminino, animais em casa no presente e ausência de peixe na dieta. A prevalência de asma ativa foi alta com redução significante à encontrada em 1998.

Asma; Adolescente; Fatores de Risco

\section{Colaboradores}

Todos os autores participaram com contribuições substanciais para a concepção e desenho, bem como para aquisição, análise e interpretação dos dados. Contribuíram também na redação do artigo e revisão crítica do conteúdo intelectual, além de darem aprovação final a versão a ser publicada.

\section{Agradecimentos}

Aos Diretores, Coordenadores e Professores das Escolas Públicas e Particulares pela disponibilidade e auxílio durante a aplicação dos questionários e realização da antropometria. Aos adolescentes e seus pais pela participação, sem a qual a realização deste trabalho não seria possível.

\section{Referências}

1. World Health Organization. Asthma. Fact sheet no 307. http://www.who.int/topics/asthma/en/ (acessado em 03/Nov/2011).

2. Global Initiative for Asthma. Global strategy for asthma management and prevention. http://www. ginasthma.org/pdf/GINA_Report_2010.pdf (acessado em 03/Nov/2011).

3. ISAAC Steering Committee. http://isaac.auckland. ac.nz/ (acessado em 02/Nov/2011).

4. Asher MI, Keil U, Anderson HR, Beasley R, Crane J, Martinez F, et al. International Study of Asthma and Allergies in Childhood (ISAAC): rationale and methods. Eur Respir J 1995; 8:483-91.

5. Strachan DP. The role of environmental factors in asthma. Br Med Bull 2000; 58:865-82.

6. Arruda LK, Solé D, Baena-Cagnanic CE, Naspitz CK. Risk factors for asthma and atopy. Curr Opin Allergy Clin Immunol 2005; 5:153-9.

7. Barros R, Moreira A, Fonseca J, Moreira P, Fernandes L, Oliveira JF, et al. Obesity and airway inflammation in asthma. J Allergy Clin Immunol 2006; 117:1501-2.

8. Castro-Rodríguez JA. Relación entre obesidad y asma. Arch Bronconeumol 2007; 43:171-5.

9. Schaub B, Von Mutius E. Obesity and asthma, what are the links? Curr Opin Allergy Clin Immunol 2005; 5:185-93.

10. Amorim AJ, Daneluzzi JC. Prevalência de asma em escolares. J Pediatr (Rio J.) 2001; 77:197-202.

11. Centers for Disease Control and Prevention. Clinical growth charts. http://www.cdc.gov/growth charts/clinical_charts.htm\#Set1 (acessado em 05/ Nov/2011).

12. Instituto Brasileiro de Geografia e Estatística. Pesquisa de Orçamentos Familiares 2002-2003. http:// www.ibge.gov.br/home/estatistica/populacao/ condicaodevida/pof/ 2003medidas/default.shtm (acessado em 15/Out/2007).

13. Asher MI, Montefort S, Björkstén B, Lai CK, Strachan DP, Weiland SK, et al. Worldwide time trends in the prevalence of symptoms of asthma, allergic rhinoconjunctivitis, and eczema in childhood: ISAAC Phases One and Three repeat multicountry cross-sectional surveys. Lancet 2007; 370:1128.

14. Pearce N, Aït-Khaled N, Beasley R, Mallol J, Keil U, Mitchell E, et al. Worldwide trends in the prevalence of asthma symptoms: phase III of the International Study of Asthma and Allergies in Childhood (ISAAC). Thorax 2007; 62:758-66.

15. Solé D, Camelo-Nunes SIC, Freitas LS, Brito M, Rosario NA, Jones M, et al. Changes in the prevalence of asthma and allergic diseases among Brazilian school children (13-14 year-old): comparison between ISAAC's Phases One and Three. J Trop Pediatr 2007; 53:13-21.

16. Rosa AM, Ignotti E, Hacon SS, Castro HA. Prevalência de asma em escolares e adolescentes em um município na região da Amazônia brasileira. J Bras Pneumol 2009; 35:7-13.

17. Farias MRC, Rosa AM, Hacon SS, Castro HA, Ignotti E. Prevalência de asma em escolares de Alta Floresta - município a sudeste da Amazônia brasileira. Rev Bras Epidemiol 2010; 13:49-57. 
18. Camelo-Nunes IC, Wandalsen GF, Melo KC, Naspitz CK, Solé D. Prevalência de asma e de sintomas relacionados entre escolares de São Paulo, Brasil: 1996 a 1999 - Estudo da reatividade brônquica entre adolescentes asmáticos e não asmáticos "International study of asthma and allergies in childhood (ISAAC). Rev Bras Alergia Imunopatol 2001; 24:77-89.

19. Casagrande RR, Pastorino AC, Souza RG, Leone C, Solé D, Jacob CM. Asthma prevalence and risk factors in schoolchildren of the city of São Paulo, Brazil. Rev Saúde Pública 2008; 42:517-23.

20. Mallol J, Aguirre V, Aguilar P, Calvo M, Amarales L, Arellano P, et al. Cambios en la prevalencia de asma en escolares chilenos entre 1994 y 2002. Rev Méd Chile 2007; 135:580-6.

21. Pastorino AC, Rimazza RD, Leone C, Castro AP, Solé D, Jacob CM. Risk factors for asthma in adolescents in a large urban region of Brazil. J Asthma 2006; 43:695-700.

22. Kuschnir FC, Cunha AJLA, Braga DAC, Silveira HHN, Barroso MH, Aires ST. Asma em escolares de 13 e 14 anos do Município de Nova Iguaçu, Rio de Janeiro, Brasil: estimativas de prevalência, gravidade e diferenças de gênero. Cad Saúde Pública 2007; 23:919-23.

23. Luna MFG, Almeida PC, Silva MGC. Prevalência e associação de asma e rinite em adolescentes de 13 e 14 anos de Fortaleza, Ceará, Brasil. Cad Saúde Pública 2011; 27:103-12.

24. Mandhane PJ, Greene JM, Cowan JO, Taylor DR, Sears MR. Sex differences in factors associated with childhood- and adolescent-onset wheeze. Am J Respir Crit Care Med 2005; 172:45-54.

25. Siroux V, Curt F, Oryszczyn MP, Maccario J, Kauffmann F. Role of gender and hormone-related events on IgE, atopy, and eosinophils in the Epidemiological Study on the Genetics and Environment of Asthma, bronchial hyperresponsiveness and atopy. J Allergy Clin Immunol 2004; 114:491-8.

26. Horner CC, Strunk RC. Age-related changes in the asthmatic phenotype in children. Curr Opin Pediatr 2007; 19:295-9.

27. Almqvist C, Worm M, Leynaert B; Working Group of GA2LEN WP 2.5 Gender. Impact of gender on asthma in childhood and adolescence: a GA2LEN review. Allergy 2008; 63:47-57.

28. Baqueiro T, Pontes-de-Carvalho L, Carvalho FM, Santos NM, Alcântara-Neves NM; Medical Student's Group. Asthma and rhinitis symptoms in individuals from different socioeconomic levels in a Brazilian city. Allergy Asthma Proc 2007; 28:362-7.

29. Shankardass K, McConnell RS, Milam J, Berhane $\mathrm{K}$, Tatalovich Z, Wilson JP, et al. The association between contextual socioeconomic factors and prevalent asthma in a cohort of Southern California school children. Soc Sci Med 2007; 65:1792-806.

30. Felizola ML, Viegas CA, Almeida M, Ferreira F, Santos MC. Prevalence of bronchial asthma and related symptoms in schoolchildren in the Federal District of Brazil: correlations with socioeconomic levels. J Bras Pneumol 2005; 31:486-91.

31. Da Cunha SS, Pujades-Rodriguez M, Barreto ML, Genser B, Rodrigues LC. Ecological study of socioeconomic indicators and prevalence of asthma in schoolchildren in urban Brazil. BMC Public Health 2007; 7:205.
32. Solé D, Camelo-Nunes IC, Wandalsen GF, Mallozi MC, Naspitz CK; Brazilian ISAAC's Group. Is the prevalence of asthma and related symptoms among Brazilian children related to socioeconomic status? J Asthma 2008; 45:19-25.

33. Bener A, Ehlayel MS, Alsowaidi S, Sabbah A. Role of breastfeeding in primary prevention of asthma and allergic diseases in a traditional society. Eur Ann Allergy Clin Immunol 2007; 39:337-43.

34. Schneider AP, Stein RT, Fritscher CC. O papel do aleitamento materno, da dieta e do estado nutricional no desenvolvimento de asma e atopia. J Bras Pneumol 2007; 33:454-62.

35. Friedman NJ, Zeiger RS. The role of breast-feeding in the development of allergies and asthma. J Allergy Clin Immunol 2005; 115:1238-48.

36. Martinez FD, Cline M, Burrows B. Increased incidence of asthma in children of smoking mothers. Pediatrics 1992; 89:21-6.

37. DiFranza JR, Aligne CA, Weitzman M. Prenatal and postnatal environmental tobacco exposure and children's health. Pediatrics 2004; 113:1007-15.

38. Feleszko W, Zawadzka-Krajewska A, Matysiak K, Lewandowska D, Peradzynska J, Dinh QT, et al. Parental tobacco smoking is associated with augmented IL-13 secretion in children with allergic asthma. J Allergy Clin Immunol 2006; 117:97-102.

39. Moshammer H, Hoek G, Luttmann-Gibson H, Neuberger MA, Antova T, Gehring U, et al. Parental smoking and lung function in children: an international study. Am J Respir Crit Care Med 2006; 173:1255-63.

40. Kurz T, Ober C. The role of environmental tobacco smoke in genetic susceptibility to asthma. Curr Opin Allergy Clin Immunol 2004; 4:335-9.

41. Meyers DA, Postma DS, Stine OC, Koppelman GH, Ampleford EJ, Jongepier H, et al. Genome screen for asthma and bronchial hyperresponsiveness: interactions with passive smoke exposure. J Allergy Clin Immunol 2005; 115:1169-75.

42. Brussee JE, Smit HA, van Strien RT, Corver K, Kerkhof M, Wijga AH, et al. Allergen exposure in infancy and the development of sensitization, wheeze, and asthma at 4 years. J Allergy Clin Immunol 2005; 115:946-52.

43. Custovic A, Simpson A. Pet and the development of allergic sensitization. Curr Allergy Asthma Rep 2005; 3:212-20.

44. Korppi M, Hyvärinen M, Kotaniemi-Syrjänen A, Piippo-Sarvolainen E, Reijonen T. Early exposure and sensitization to cat and dog: different effects on asthma risk after wheezing in infancy. Pediatr Allergy Immunol 2008; 19:696-701.

45. Devereux G, Seaton A. Diet as a risk factor for atopy and asthma. J Allergy Clin Immunol 2005; 115:1109-17.

46. Visness CM, London SJ, Daniels JL, Kaufman JS, Yeatss KB, Siega-Riz AM, et al. Association of childhood obesity with atopic and nonatopic asthma: results from the National Health and Nutrition Examination Survey 1999-2006. J Asthma 2010; $47: 822-9$

Recebido em 11/Ago/2011

Versão final reapresentada em 03/Dez/2011

Aprovado em 15/Dez/2011 